\title{
LA RELACIÓN ENTRE LA IMPULSIVIDAD COGNITIVA (R-I) Y EL MALTRATO ENTRE IGUALES O "BULLYING" EN EDUCACIÓN PRIMARIA
}

\author{
M* Carmen Plchardo Martínez \\ José Luls Arco Tirado \\ Francisco D. Fernández Martín
}

Departamento de Psicología Evolutiva y de la Educación. Universidad de Granada

\section{RESUMEN}

El propósito del estudio fue conocer la relación existente entre el fenómeno "bullying" y la impulsividad cognitiva. El alumnado participante pertenecia a un Colegio Público de Educación Primaria de la provincia de Granada, concretamente de sexto curso. Para llevar a cabo el estudio, se utilizó el Cuestionario sobre abusos entre compañeros de Femández y Ortega (1998), Técnicas de Observación, a través de un Sistema Verbal de Codificación (Registros Diarios de Heterobservación), y el MFF20 de Caims y Cammock (1978). Los resultados manifestaron que el alumnado que desempeña el rol de "agresor" en el fenómeno "bullying" suele ser impulsivo a nivel cognitivo en relación al alumnado que desempeña un rol diferente ya que presenta mayor media en errores y menor media en latencias.

Palabras clave: ESTLLOS COGNITIVOS, REFLEXIVIDAD-IMPULSIVIDAD, MALTRATO ENTRE COMPAÑEROS, EDUCACIÓN PRIMARIA. 


\section{SUMMARY}

The purpose of this study was to find out about the relationship between the phenomenon of "bullying" and cognitive impulsivity. All subjects participating on this study were students of six grade at Primary Public School in Granada. Several instruments were used like the Questionnaire of Abuse Among Peer Mates by Fernández \& Ortega (1998), Observation Techniques by a codification of a verbal system (Heterobservation Daily Registers) and the MFF20 by Cairns \& Cammock (1978). The results show that those students who performed the roll of "aggressor", within the bulling phenomenon, tends to be cognitively impulsive compared to those students under other roles, since they presented higher average on response errors and lower average on latencies.

Key words: COGNITVE STYLES, REFLECTON-IMPULSIVITY, BULLYING, PRIMARY EDUCATON.

La investigación sobre la reflexividad-impulsividad (R-I) 0 impulsividad cognitiva proviene de los estudios sobre los estilos cognitivos que surgieron de la necesidad de establecer un puente entre ciertas dimensiones cognitivas relacionadas con la percepción y la personalidad (Carretero y Palacios, 1982; Garcia, 1989). Este constructo bipolar, que va de la rapidez y un elevado número de errores (impulsividad) a la lentitud y un porcentaje bajo de errores (reflexividad) (Kagan, 1965a, 1965b), posee numerosas implicaciones educativas, que indican que el alumnado reflexivo obtiene mejores puntuaciones en la inhibición de la respuesta y control de movimientos (Harrison y Nadelman, 1972), la metacognición (Cameron, 1984), la solución de problemas (Messer, 1976; Zakay, Bar-El y Kreitler, 1984), el dominio de la agresividad y el autocontrol (Mann, 1973; Ward, 1973) y la conducta moral y el juicio moral (Schleifer y Douglas, 1973), dimensiones estas que influyen notablemente en el comportamiento del alumnado.

Por tanto, tomando como base estas implicaciones de la R-I, se puede llegar a pensar que la impulsividad cognitiva ha de jugar un 
papel importante en el maltrato entre compañeros o "bullying", ya que el alumnado reflexivo, sí puntúa mejor en los aspectos mencionados anteriormente, tendrá una menor tendencia que el alumnado impulsivo a implicarse en conductas violentas o de "bullying" contra otros compañeros. No obstante, la literatura sobre esta temática, que a continuación se tratará, no deja de ser suficientemente problemática y controvertida debido a la presencia de aportaciones contrapuestas de diferentes autores.

Por un lado Buela, Carretero y De los Santos (2002), cuando describen la relación entre R-l y el comportamiento del alumnado ante situaciones conflictivas con sus iguales, defienden que el alumnado impulsivo tiende a poner en marcha conductas de evitación o sumisión ante cualquier situación de estas características, y el reflexivo, es el que opta por tomar un papel activo en estas situaciones, caracterizado por una postura asertiva o violenta, predominando ésta última debido a las influencias del modelado social. Así, desde esta perspectiva, el alumnado impulsivo a nivel cognitivo estaría más identificado con el rol de "víctima", o "espectador", en el fenómeno "bullying", mientras que el reflexivo se identificaría más con el rol de "agresor" en el caso de que optara por un papel activo-violento, que es lo predominante. Es decir, el alumnado reflexivo es el que tiende generalmente a la violencia.

Los estudios mencionados anteriormente sobre la R-I, manifiestan en cambio, que el alumnado reflexivo supera positivamente al impulsivo en aspectos que influyen notablemente en el comportamiento, por lo que es difícil coincidir con la postura inicial de Buela, Carretero y De los Santos (2002). Es decir, si el alumnado reflexivo es mejor que el impulsivo a la hora de autocontrolarse, dominar la agresividad, inhibir la respuesta y control de movimientos, etc., entonces difícilmente va a ser más violento. En otras palabras, que el alumnado impulsivo, generalmente, tiende más a la violencia que el reflexivo.

En esta línea, Messer y Brodzinsky (1979), en un estudio en el que exploraban las relaciones entre R-l y agresividad, encontraron que los niños impulsivos eran más agresivos, especialmente al compararlos con los reflexivos. Según estos autores parece que el niño impulsivo está menos socializado y se siente más libre para expresar sus impulsos sin preocuparse de las consecuencias. Además, existen 
otras investigaciones con poblaciones que presentaban dificultades que concluyen de forma similar, como es el caso de los trabajos de Campbell y Douglas (1972), Finch y Nelson (1976), Montgomery y Finch (1975) y Weintraub (1973).

Por otro lado, existen algunas investigaciones que asignan características de personalidad a los grupos reflexivos e impulsivos que surgen como fruto de la evaluación llevada a cabo a través del Test de Emparejamiento de Figuras Familiares (MFFT) de Kagan (1965c). Así, el alumnado evaluado como impulsivo mediante este instrumento, se caracteriza por ser: agresivo, competitivo, poco sensible a los demás, baja tolerancia a las restricciones, rápidos, no inhibidos, expresa directamente sus frustraciones y conflictos y tienen dificultad para postergar las gratificaciones; y el reflexivo por ser: razonable, reflexivo, tranquilos, considerados, conciliadores, inteligentes y esforzados (Block, Block y Harington, 1974). No obstante, estos estudios poseen algunas deficiencias metodológicas, entre las que destaca la muestra (población de preescolar), y la carencia de instrumentos lo suficientemente válidos y fiables para medir este fenómeno en estas edades, por lo que los resultados hemos de tomarlos con cautela.

En otra línea se posicionan otros autores que defienden que el alumnado impulsivo, a nivel cognitivo, puede ser o no ser violento, ya que nada garantiza una relación causal ni correlacional (Servera y Galván, 2001). Para afirmar esto, se basan en la diferenciación de los tres tipos de impulsividad (cognitiva, manifiesta y personológica) y la relación que se da entre ellas: "la impulsividad cognitiva comparte con las otras dos la idea de precipitación y falta de consideración de las consecuencias, pero se diferencia de la manifiesta en que no tiene por qué ir ligada a problemas de control motor, y de la personológica, en que no tiene por qué demostrarse en el espectro de funcionamiento social de la persona" (Servera y Galván, 2001, p. 14). Es decir, un niño impulsivo a nivel cognitivo no tiene porque tener problemas de control motor ni influirle estos en su actividad social.

Así pues, las diversas aportaciones concluyen de manera contradictoria que: el alumnado reflexivo es el que tiende generalmente a la violencia (Buela, Carretero y De los Santos, 2002); el alumnado impulsivo, generalmente, tiende más a la violencia que el reflexivo 
(Messer y Brodzinsky, 1979; Campbell y Douglas, 1972; Weintraub, 1973; Finch y Nelson, 1976; Montgomery y Finch, 1975); el alumnado impulsivo sería agresivo (Block, Block y Harrington, 1974); y por último, que el alumnado impulsivo no tiene porque tener problemas de control motor ni influirle estos en su actividad social (Servera y Galván, 2001). No obstante, aquí de nuevo hemos de ser prudentes en la validez de las conclusiones debido a los problemas metodológicos que presentan algunas de estas investigaciones. La única que parece sustentarse por su rigor metodológico es la segunda; es decir, aquella que defiende que el niño impulsivo, debido a sus características, puede tener una mayor tendencia hacia conductas violentas.

En referencia a la cuarta aportación (Servera y Galván, 2001), la precipitación y falta de consideración de las consecuencias ante una situación con cierto grado de incertidumbre, nexo entre las tres impulsividades, viene dada por un procesamiento concreto de la información que influye a nivel académico, pero que, según estos autores, no tiene porque incidir en las relaciones interpersonales, así como en otros contextos. Sin embargo, esa relación que se da entre las tres impulsividades de una $u$ otra forma ha de incidir en las demás situaciones y contextos en los que el alumnado se desenvuelve. Tanto es así, que Haskins y Mckinney (1976), observando a niños reflexivos e impulsivos en diferentes tareas escolares encontraron notables diferencias en la conducta del alumnado. Además, las investigaciones recientes en materia de "bullying" apuntan a que la conducta violenta es el resultado de una inadaptación debida a problemas en el procesamiento de la información que dificultan la elaboración de respuestas alternativas, por lo que el niño violento se muestra menos reflexivo y considerado hacia los sentimientos, pensamientos e intenciones de los demás (Cerezo, 2002). A su vez, los estudios descriptivos de este fenómeno caracterizan al alumnado que desempeña el rol de agresivo como: impulsivo o poco reflexivo, con dificultad para situarse en la perspectiva de otro (empatia), con falta de autocontrol, de carácter difícil, con bajo rendimiento académico, etc. (Cerezo, 2002; Justicia, 2001; Olweus, 1993).

Por tanto, podríamos concluir que el alumnado que desempeña el rol de "agresor" en el fenómeno "bullying" suele ser, generalmente, impulsivo a nivel cognitivo, y va a ser más violento que el reflexivo. 
Así pues, el objetivo general de esta investigación fue conocer cómo influye en el fenómeno "bullying" la impulsividad cognitiva en una población de educación Primaria, planteándose para ello como hipótesis que los alumnos de la muestra analizada cuyo rol en el fenómeno "bullying" es "agresor" presentan niveles de impulsividad cognitiva superiores a los alumnos con roles diferentes.

\section{MÉTODO}

\section{Muestra}

La muestra de este estudio estuvo compuesta por 76 alumnos de sexto curso de Primaria de un Colegio Público del área metropolitana de Granada. Este grupo de alumnos poseía unas edades que oscilaban entre los 10 y los 12 años, con una media de 10 años y once meses. La distribución de la muestra por edades y sexo queda reflejada en la tabla 1.

Tabla 1. Descripción de la muestra

\begin{tabular}{cccc}
\hline & & Frecuencia & Porcentaje \\
\hline \multirow{3}{*}{ Edad } & 10 años & 5 & 6,58 \\
& 11 años & 67 & 88,16 \\
& 12 años & 4 & 5,26 \\
& Total & 76 & 100,00 \\
Sexo & Hombre & 40 & 52,63 \\
& Mujer & 36 & 47,37 \\
& Total & 76 & 100,00 \\
\hline
\end{tabular}

\section{Variables e instrumentos}

Para el análisis de las relaciones de malos tratos entre iguales ("bullying") así como para conocer el grado de reflexividad e impulsividad de los participantes se utilizaron los siguientes instrumentos:

a) "Cuestionario sobre abusos entre compañeros" de Fernández y Ortega (1998). Se decidió utilizar este instrumento por ser uno de los 
pocos que mide el fenómeno objeto de evaluación en el alumnado de Educación Primaria, además de adecuarse al contexto. Esta prueba es de carácter anónimo $\theta$ indaga las percepciones sobre las situaciones relacionadas con los abusos o malos tratos entre escolares. Se puede administrar en grupo o individualmente y consta de 25 ítems de opción múltiple agrupados en dos grandes grupos, uno que versa sobre la vida relacional del alumnado ( 8 ftems), y otro, sobre las situaciones de abuso entre compañeros (17 items).

b) "Técnicas de Observación", a través de un Sistema Verbal de Codificación. Se utilizaron Registros Diarios de Heteroobservación. El uso de este instrumento viene justificado por la carencia de estudios de validez y fiabilidad del Cuestionario sobre abusos entre compañeros de Fernández y Ortega (1998). Su objetivo fue recoger observaciones sobre las conductas "bullying" (quién las realiza, quién las sufre y quién las observa) que se daban en el patio del recreo y en la clase, con la finalidad de clasificar al alumnado según el rol que desempeñe en el fenómeno (Agresor, Víctima, Víctima-agresor y Espectador). Para ello, se siguieron todos los criterios que definen el fenómeno y las características de sus diferentes roles. Estos registros se llevaron a cabo durante dos meses a través de un muestreo intrasesional multifocal o de barrido, utilizando una regla de registro activado por transiciones.

c) "Test de Emparejamiento de Figuras Familiares" (MFF20) de Cairns y Cammock (1978). La utilización de este instrumento viene justificada por ser la prueba que comúnmente se utiliza para evaluar el continuo reflexividad-impulsividad debido a su mayor validez (correlación errores-latencias alrededor de $-0,68$ ) y fiabilidad (por consistencia interna de los errores sobre 0,70 y de las latencias sobre 0,94). El MFF20 es un test de emparejamiento perceptivo que consta de 20 ítems más otros dos de prueba, cada uno de los cuales presenta simultáneamente al sujeto un dibujo estándar o modelo y seis variantes o copias casi idénticas del mismo, de las cuales sólo una es exactamente igual al modelo. El sujeto tiene que averiguar cuál es. Se anota el tiempo que tarda en dar la primera respuesta y el número de errores que comete al resolverlo, para más tarde calcular la media y desviación estándar de las puntuaciones de errores y latencias. 


\section{Procedimlento}

Este estudio, que surge como fruto del análisis de necesidades llevado a cabo en un Centro Educativo de la provincia de Granada, se desarrolló a través de dos fases: análisis de necesidades, para conocer y priorizar las necesidades de la muestra, y una segunda fase de evaluación, para determinar la incidencia de las necesidades.

El análisis de necesidades se realizó, fruto de la demanda que formularon los docentes y padres al Equipo de Orientación Educativa (EOE) de la zona, a través de una serie de seminarios/reuniones de unas tres horas de duración. En estos seminarios/reuniones, donde participaron docentes, padres, investigadores, miembros del EOE y de los diferentes equipos directivos, se trataron aspectos de diversa índole relacionados con el ambiente social, educativo y económico del centro, las familias y la comunidad.

A partir de estos seminarios/reuniones se dieron a conocer una serie de necesidades relacionadas con el alumnado de sexto curso de educación Primaria, destacando sobre todo la presencia de dificultades de atención e impulsividad a la hora de realizar cualquier tarea, actividad y/o conducta, y ciertas dificultades en referencia a las relaciones interpersonales.

Una vez determinadas y priorizadas las necesidades, se pasó a recoger los valores que la muestra presentaba en los problemas y variables implicadas, lo que permitió determinar su frecuencia o incidencia.

En un primer lugar se aplicó por parte de uno de los investigadores el MFF20 de Cairns y Cammock (1978) a lo largo de 76 sesiones individuales, con la condición de que fuera el sujeto el que decidiera cómo responder ante la incertidumbre, es decir, no se le instruía para que respondiera rápido o despacio, ni tampoco se le indicaba que procurara ser exacto en sus respuestas. La estructura de las sesiones viene dada por una breve presentación del investigador y la explicación de las instrucciones, para después pasar a los dos primeros ftems, que son de ejemplo, asegurando así la comprensión de la tarea. Más tarde, se administran los demás f́tems tomando nota del tiempo que tarda el alumnado en dar la primera respuesta a cada ítem de los que siguen a los dos de prueba y del número de errores que comete al resolverlo. 
En un segundo momento, se aplicó por parte del mismo investigador el Cuestionario sobre abusos entre compañeros de Fernández y Ortega (1998) a lo largo de tres sesiones grupales (grupo-clase) de evaluación de aproximadamente 45 minutos de duración. La administración de este cuestionario se precedía de un debate con el grupo de unos 10 minutos, en el que se pretendía aclarar al alumnado el significado de las situaciones de maltrato y/o abuso entre compañeros, y de la explicación de las instrucciones, para después pasar a rellenar el cuestionario.

Por otro lado, el desarrollo de los Registros Diarios de Heteroobservación fue llevado a cabo por el investigador a lo largo de 60 sesiones en la clase de 35 minutos de duración y 60 sesiones en el recreo de 25 minutos de duración durante dos meses. La observación y registro se centró en la presencia de las conductas "bullying", quién las realizaba, quién las sufría y quién las observaba.

Para finalizar, una vez analizados los resultados, se concertó una reunión en el Centro Educativo para comunicar y discutir los resultados obtenidos.

\section{Análisis de Datos}

Debido a que la información recopilada a través de los distintos instrumentos era tanto de naturaleza cuantitativa como cualitativa, se utilizaron diversos procedimientos para llevar a cabo el análisis de datos.

Así, la información recogida a través del Cuestionario sobre abuso entre compañeros (Fernández y Ortega, 1998), cuyo objetivo era conocer la incidencia del fenómeno "bullying" en el centro escolar, fue analizada con el paquete estadístico SPSS-12.0. Este análisis descriptivo se centró en el cálculo de frecuencias y porcentajes.

Por otro lado, para conocer los diferentes roles dentro del fenómeno "bullying" que desempeñaba la muestra, se usó la información procedente del cuestionario anteriormente citado y de los Registros Diarios de Heteroobservación. Para ello, en el caso del Cuestionario de Fernández y Ortega (1998), después de consultar con expertos, se centró la atención en la respuesta del alumnado a una serie de ítems que caracterizan cada uno de los roles del "bullying" (víctimas: 7, 8, 9, $10,11,12,13,14,15,16,17$ y 18 ; agresores: 19,20 y 25 ; espectadores: $21,22,23$ y 24), realizándose un análisis descriptivo de las respuestas 
dadas por cada sujeto. Por su parte, para analizar la información procedente del material de los Registros Diarios de Heteroobservación, se realizó un análisis de contenidos: se delimitaron las unidades de análisis para su categorización (sistema de categorias: conductas que definen cada uno de los roles). Para tinalizar se cré́ una auditoria con toda la información y datos, en la que estaban presentes el investigador, algunos expertos y los docentes implicados, donde se triangulo la información y se categorizó a cada alumno en un rol.

A su vez, la información procedente del MFF20 de Cairns y Cammock (1978), también fue analizada a través del paquete estadístico SPSS-11. Sobre los datos resultantes de la prueba se realizaron los siguientes análisis: (a) estadisticos descriptivos para conocer las medidas de tendencia central para la muestra; (b) medidas de asociación direccionales y simétricas para las variables sexo y rol desempeñado en el fenómeno "bullying", con el objetivo de comprobar la existencia de relación entre las dos variables; (c) análisis de varianza, con la finalidad de detectar la presencia de diferencias estadísticamente significativas en errores y latencias entre los diferentes roles (agresor, victima, victima-agresor y espectador) (comparaciones múltiples) y en errores y latencias entre sexos.

\section{RESULTADOS}

En cuanto a los resultados relacionados con el fenómeno del maltrato entre compañeros ("bullying") tal y como se observa en la tabla 2 , un $19,74 \%$ de este alumnado puede categorizarse como "agresor" (alumnado que lleva a cabo las agresiones sobre otros compañeros), un 19,74\% como "víctima" (alumnado que es el blanco de las agresiones producidas por el alumnado anterior), un $9,21 \%$ como "víctima-agresor"(alumnado que comparte características tanto de las victimas como de los agresores) y un $51,31 \%$ como "espectador" (alumnado que asiste y observa las conductas "bullying", sin ser el agresor o la víctima).

Por otra parte, los resultados del análisis de R-I arrojan, que el grupo de participantes en la investigación posee una media de errores de 3,81 con una desviación típica de 3,19. En cuanto a las latencias, se presenta una media de 573,00 segundos con una desviación típica de 184,85. 
Tabla 2. Categorización de la muestra según su rol en las situaciones de abuso/victimización

\begin{tabular}{lcc}
\hline \multicolumn{1}{c}{ Roles } & N & $\%$ \\
\hline Víctimas & 15 & 19,74 \\
Agresores & 15 & 19,74 \\
Víctima-agresor & 7 & 9,21 \\
Espectadores & 39 & 51,31 \\
\multicolumn{1}{c}{ TOTAL } & 76 & 100,00 \\
\hline
\end{tabular}

Al relacionar el fenómeno "bullying" y la impulsividad cognitiva observamos (Tabla 3) que, en cuanto a la media de errores, los participantes categorizados como "agresores" poseen la media más elevada, seguidos de los participantes categorizados como "víctimas-agresores", siendo las "víctimas" y los "espectadores" los que obtienen medias de errores más bajas. En relación con las medias obtenidas en latencia los datos reflejan que en este caso son los participantes "victimas" y los "espectadores" los que reflejan una mayor latencia de respuesta frente a los "agresores" y "víctimas-agresores" que serían los que obtienen medias de latencia más bajas.

Realizadas las medidas de asociación direccionales y simétricas para las variables sexo y rol desempeñado en el fenómeno "bullying" no se aprecian diferencias estadisticamente significativas $(T=0,68$; $p>0,05$ ) entre ambas.

Por su parte, la prueba de análisis de varianza efectuada (Tabla 3) arroja diferencias estadísticamente significativas en errores entre los diferentes roles de la muestra $(F=8,59 ; p<0,05)$. Igualmente, se producen diferencias estadísticamente significativas con relación al tiempo de latencia de respuesta entre los diferentes roles $(F=6,85$; $p<0,05)$, a diferencia de los resultados encontrados para las variables sexo y media de errores $(F=0,09 ; p>0,05)$, y sexo y media de latencias ( $F=0,02 ; p>0,05)$.

Los resultados de las comparaciones múltiples entre los diferentes roles tanto en errores como en latencias, establecen la existencia de diferencias significativas entre los roles de "agresor" y "victima" $(p<0,05)$ y entre "agresor" y "espectador" $(p<0,05)$ en errores. Los alumnos categorizados como "víctimas" y "espectadores" obtienen 
Tabla 3. Análisis de varianza de los roles desempeñados en el fenómeno bullying con relación a los errores y latencias

\begin{tabular}{|c|c|c|c|c|c|c|}
\hline Variable & Ral & $\mathbf{N}$ & Media & Desv. típ. & $\mathbf{F}$ & Sig. \\
\hline \multirow{5}{*}{ Errores } & Agresor & 15 & 6,73 & 3,08 & \multirow{5}{*}{8,59} & \multirow{5}{*}{0,001} \\
\hline & Víctima & 15 & 2,87 & 2,39 & & \\
\hline & Víctima-Agresor & 7 & 4,71 & 2,69 & & \\
\hline & Espectador & 39 & 2,72 & 2,73 & & \\
\hline & Total & 76 & 3,72 & 3,13 & & \\
\hline \multirow{4}{*}{ Latencias } & Agresor & 15 & 411,07 & 128,04 & \multirow{5}{*}{6,85} & \multirow{5}{*}{0,001} \\
\hline & Victima & 15 & 657,27 & 137,86 & & \\
\hline & Víctima-Agresor & 7 & 517,71 & 259,35 & & \\
\hline & Espectador & 39 & 608,56 & 170,13 & & \\
\hline \multirow{4}{*}{ Latencias } & Total & 76 & 570,83 & 185,09 & & \\
\hline & Varón & 40 & 573,60 & 174,43 & \multirow{3}{*}{0,02} & \multirow{3}{*}{0,892} \\
\hline & Mujer & 36 & 567,75 & 198,72 & & \\
\hline & Total & 76 & 570,83 & 185,09 & & \\
\hline \multirow{3}{*}{ Errores } & Varón & 40 & 3,40 & 3,39 & \multirow{3}{*}{0,90} & \multirow{3}{*}{0,345} \\
\hline & Mujer & 36 & 4,08 & 2,81 & & \\
\hline & Total & 76 & 3,72 & 3,13 & & \\
\hline
\end{tabular}

medias en errores significativamente más bajas que los alumnos que se les categorizó como "agresores".

En cuanto a las latencias, se encuentran igualmente diferencias significativas entre los roles de "agresor" y "víctima" $(p<0,05)$ y entre "agresor" y "espectador" ( $p<0,05)$, obteniendo medias más altas en latencia de respuesta las "víctimas" y "espectadores", que los "agresores".

Por el contrario, no se encuentran diferencias significativas en errores y latencias entre los alumnos "agresores" y los "victimasagresores", ni tampoco entre estos últimos y los "victimas" o "espectadores", considerándose por tanto similares sus tiempos de latencia y sus tasas de errores.

\section{DISCUSIóN}

De acuerdo a nuestros resultados, el porcentaje de alumnos implicados, de forma severa y/o moderada, en el fenómeno "bullying" es del 48,69\% ("agresores" 19,74\%; "víctimas" 19,74\%; "víctimas- 
Tabla 4. Comparaciones múltiples entre los diferentes roles en errores y latencias

\begin{tabular}{|c|c|c|c|c|}
\hline Variables $\mathrm{R} / \mathrm{I}$ & Rol & Rol & Dif. Med. & Sig. \\
\hline \multirow{11}{*}{ Errores } & \multirow{4}{*}{ Agresor } & Víctima & 3,87 & 0,003 \\
\hline & & Víctima-Agresor & 2,02 & 0,463 \\
\hline & & Espectador & 4,02 & 0,0001 \\
\hline & & Agresor & $-3,87$ & 0,003 \\
\hline & \multirow[t]{2}{*}{ Victima } & Victima-Agresor & $-1,85$ & 0,541 \\
\hline & & Espectador & 0,15 & 0,998 \\
\hline & \multirow{4}{*}{ Victima-Agresor } & Agresor & $-2,02$ & 0,463 \\
\hline & & Victima & 1,85 & 0,541 \\
\hline & & Espectador & 2,00 & 0,375 \\
\hline & & Agresor & $-4,02$ & 0,0001 \\
\hline & \multirow[t]{3}{*}{ Espectador } & Victima & $-0,15$ & 0,998 \\
\hline \multirow{13}{*}{ Latencia } & & Victima-Agresor & $-2,00$ & 0,375 \\
\hline & & Victima & $-246,20$ & 0,002 \\
\hline & \multirow[t]{3}{*}{ Agresor } & Victima-Agresor & $-106,65$ & 0,584 \\
\hline & & Espectador & $-197,50$ & 0,003 \\
\hline & & Agresor & 246,20 & 0,002 \\
\hline & \multirow[t]{3}{*}{ Victima } & Victima-Agresor & 139,55 & 0,348 \\
\hline & & Espectador & 48,70 & 0,819 \\
\hline & & Agresor & 106,65 & 0,584 \\
\hline & \multirow[t]{3}{*}{ Víctima-Agresor } & Victima & $-139,55$ & 0,348 \\
\hline & & Espectador & $-90,85$ & 0,625 \\
\hline & & Agresor & 197,50 & 0,003 \\
\hline & \multirow[t]{2}{*}{ Espectador } & Victima & $-48,70$ & 0,819 \\
\hline & & Victima-Agresor & 90,85 & 0,625 \\
\hline
\end{tabular}

agresores" 9,21\%). Estos resultados, manifiestan un indice de frecuencia significativamente superior a los resultados obtenidos por otras investigaciones en el ámbito nacional e internacional (18,3\%, Ortega y Mora-Merchán, 1997; 16\%, Olweus, 1993; 14\%, Whitney y Smith, 1993), reafirmando asi la falta de homogeneidad que presentan la gran mayoría de las investigaciones realizadas sobre la incidencia de este problema, lo que origina la imposibilidad de comparar los resultados obtenidos en este estudio con la gran mayoría de los hasta ahora realizados. Esta falta de homogeneidad viene ocasionada por la utilización de diferentes cuestionarios, asi 
como procedimientos, grupos de edad estudiados, diseños y análisis estadisticos (Defensor del Pueblo, 2000). Aún así, a pesar de todo, los resultados del estudio son bastante similares a los obtenidos por Viera, Fernández y Quevedo (1989) y Fernández y Quevedo (1991), quiénes utilizaron un instrumento parecido con una muestra de edades comprendidas entre los 8 y 12 años (1200 alumnos), ya que el $17,3 \%$ de la muestra manifiesta intimidar y el $17,2 \%$ afirma haber sido intimidado $(34,5 \%$ implicados, sin hacer referencia a las "víctimasagresores"). En este sentido, Prados y Díaz-Atienza (2001), utilizando el mismo instrumento y con una muestra de similares características a la que ha participado en este estudio, aunque superior en número, encontraron que un tercio de la población manifestaba haber sido intimidada y más de un tercio haber intimidado.

Así pues, una vez identificados los roles que desempeñaba el alumnado de la muestra en el fenómeno "bullying", se puede centrar la discusión en conocer cómo influye en el fenómeno "bullying" la impulsividad cognitiva, a través de la media en errores y latencias de cada uno de los roles, descartando el efecto de la variable sexo tanto en relación a R-I como a los diferentes roles del fenómeno "bullying".

El alumnado de la muestra que desempeñaba un rol de "agresor" dentro del fenómeno "bullying", presenta una media mayor de errores y una media menor de latencias que el alumnado cuyo rol en este fenómeno era "víctima-agresor", "espectador" o "víctima", incrementándose la media de errores y disminuyendo la media de latencias sobre todo al compararlo con estos dos últimos roles. Así pues, teniendo en cuenta que la reflexividad-impulsividad, impulsividad cognitiva o del procesamiento de la información es un constructo bipolar que va de la rapidez y un elevado número de errores (impulsividad) ante una situación con cierto grado de incertidumbre, a la lentitud y un porcentaje bajo de errores (reflexividad) (Kagan, 1965a, 1965b), se puede afirmar que, en la muestra participante, el alumnado "agresor" suele ser impulsivo a nivel cognitivo con relación al alumnado que desempeña otro rol diferente ya que presenta mayor media de errores y menor media de latencias, corroborándose así la hipótesis planteada.

Sobre esta cuestión, investigaciones recientes del fenómeno "bullying" apuntan a que la conducta violenta es el resultado de una 
inadaptación debida a problemas en el procesamiento de la información que dificultan la elaboración de respuestas alternativas, por lo que el niño violento se muestra menos reflexivo y considerado hacia los sentimientos, pensamientos e intenciones de los demás (Cerezo, 2002). Además, los estudios descriptivos de este fenómeno caracterizan al alumnado que desempeña el rol de "agresivo" como impulsivo o poco reflexivo, con dificultad para situarse en la perspectiva de otro (empatía), con falta de autocontrol, de carácter difícil, etc. (Olweus, 1993; Cerezo, 2002; Justicia, 2001). También, numerosos estudios centrados en la R-I que han analizando las relaciones entre R-I y agresividad, concluyen señalando que los niños impulsivos suelen, generalmente, ser más violentos, sobre todo comparándolos con los reflexivos (Messer y Brodzinsky, 1979; Campbell y Douglas, 1972; Weintraub, 1973; Finch y Nelson, 1976; Montgomery y Finch, 1975).

Asi pues, podríamos concluir que el alumnado de la muestra que desempeña el rol de "agresor" en el fenómeno "bullying", suele ser impulsivo a nivel cognitivo, sobre todo al compararlo con el alumnado que desempeña un rol diferente, especialmente con los alumnos que desempeñan los roles de "víctima" y "espectador".

No obstante somos conscientes de que al igual que la gran mayoría de estudios realizados, esta investigación presenta limitaciones que pueden afectar a los resultados obtenidos, y por tanto, también a su generalización. Estas limitaciones serían: las limitaciones conceptuales del constructo R-I utilizado; el tamaño de la muestra; los instrumentos utilizados, ya que el Cuestionario de Fernández y Ortega (1998) carece de datos sobre su fiabilidad y validez y el MFF20 de Cairns y Cammock (1978) posee diversos problemas a nivel psicométrico.

En consecuencia, a partir de los resultados de este estudio, se hace necesario comprobar si la intervención en reflexividad, con la finalidad de inhibir la impulsividad cognitiva, incide en la reducción de las situaciones de maltrato entre compañeros o "bullying" potenciando los cambios de rol. Por tanto, de cara a futuras investigaciones, la reflexividad habria de ser tenida en cuenta como un componente más de prevención del maltrato entre iguales, de cara al diseño y posterior puesta en práctica de la intervención en los participantes que componen esta muestra. 


\section{REFERENCIAS BIBLIOGRÁFICAS}

Anguera, M. T. (Ed.) (1993). Metodologia Observacional en la Investigación Psicológica. Vol. 1 Fundamentación. Barcelona: PPU. Anguera, M. T., Arnau, J., Ato, M., Martínez, R., Pascual, J., y Vallejo, G. (1995). Métodos de Investigación en Psicologia. Madrid: Síntesis.

Block, J., Block, J. H., y Harrington, D. M. (1975). Comment on the Kagan-Messerreply. Developmental Psychology, 11, 249-252.

Buela, G, Carretero, H., y De los Santos, M. (2000). Reflexividad frente a impulsividad en el rendimiento académico: un estudio longitudinal. Análisis y Modificación de Conducta, 26, 555-583.

Buela, G, Carretero, H., y De los Santos, M. (2002). El Niño Impulsivo. Estrategias de Evaluación, Tratamiento y Prevención. Madrid: Pirámide.

Cairns, F. D., y Cammock, J. (1978). Development of a more reliable version of the matching familiar figures test. Developmental Psychology, 5, 555-560.

Cameron, R. (1984). Problem solving inefficiency and conceptual tempo: a task analysis of undenlying factors. Child Development, 55, 2031-2041.

Campbell, S. B., y Douglas, V. I. (1972). Cognitive Styles and responses to the threat of frustration. Canadian Joumal of Behavioural Sciences, 4, 30-42.

Carretero, M., y Palacios, J. (1982). Los estilos cognitivos: introducción al problema de las diferencias cognitivas individuales. Infancia $y$ Aprendizaje, 17, 20-28.

Cerezo, F. (2002). Conductas Agresivas en la Edad Escolar. Aproximación Teórica y Metodológica. Propuesta de Intervención. Madrid: Pirámide.

Defensor del Pueblo (2000). Informe sobre Violencia Escolar. Madrid: Oficina del defensor del Pueblo.

Fernández, I. (1998). Prevención de la Violencia y Resolución de Conflictos. El Clima como Factor de Calidad. Madrid: Narcea.

Femández, I., y Ortega, R. (1998). Cuestionario sobre abusos entre compañeros. En I. Fernández (Coord.), Prevención de la Violencia y Resolución de Conflictos (pp. 210-214). Madrid: Narcea. 
Fernández, I., y Quevedo, G. (1991). Como te chives ya veras. Cuadernos de Pedagogía, 193, 69-72.

Finch, A. J., y Nelson, W. M. (1976). Reflection-impulsivity and behavioral in emotionally disturbed boys. Joumal of Genetic Psychology, 128, 271-274.

García, J. M. (1989). Los Estilos Cognitivos y su Medida: Estudios sobre la Dimensión Dependencia-Independencia de Campo. Madrid: CIDE.

Gargallo, B., y García, R. (1996). La promoción del desarrollo moral a través del incremento de reflexividad. Un programa pedagógico. Revista de Educación, 309, 287-308.

Harrison, A., y Nadelman, L. (1972). Conceptual tempo and inhibition of movement in black presschool children. Child Development, 43, 657-668.

Haskins, A., y Mckinney, J. D. (1976). Relative effect of response tempo and accuracy on problem solving and academic achievement. Child Development, 47, 690-696.

Justicia, F. (2001). La convivencia en los centros educativos. En J. A. Amezcua, F. Peñafiel, y D. González (Coords.), El Psicopedagogo en el Desarrollo Comunitario. La Planificación de los Recursos Humanos (pp. 131-143). Granada: Grupo Editorial Universitario.

Kagan, J. (1965a). Individual differences in the resolution of response uncertainty. Journal of Personality and Social Psychology, 2, 154160.

Kagan, J. (1965b). Information processing in the child. En P. M. Mussen, J. J. Conger, y J. Kagan (Eds.), Readings in Child Development and Personality (pp. 284-304). New York: Harper and Row.

Kagan, J. (1965c). Matching Familar Figures Test. Cambridge: Harvard University.

Kogan, N. (1971). Educational implications of cognitive styles. En G S. Lesser (Dir.), Psychology and Educational Practice (pp. 242-292). Glenview: Scott Foresman.

Mann, L. (1973). Difference between reflective and impulsive children in ternpo and quality of decision making. Child Development, 44, 274-279.

Messer, S. B. (1976). Reflection-impulsivity: a review. Psychological Bulletin, 83(6), 1026-1052. 
Messer, S. B., y Brodzinsky, D. M. (1979). The relation of conceptual tempo to aggression and its control. Child Dovelopment, 50, 758766.

Montgomery, L. E., y Finch, A. J. (1973). Reflection-impulsivity and locus of conflict in emotionally disturben children. Joumal of Genetic Psychology, 126, 89-92.

Olweus, D. (1993). Bullying at School. What We Know and What We Can Do. Cambridge: Black-well.

Olweus, D. (1998). Conductas de Acoso y Amenazas Entre Escolares. Madrid: Morata.

Ortega, R., y Mora-Merchán, J. A. (1997). Agresividad y violencia. El problema de la victimización entre iguales. Revista de Educación, 313, 7-27.

Palacios, J. (1982). Reflexividad-impulsividad. Infancia y Aprendizaje, 17, 29-69.

Prados, M., y Díaz-Atienza, F. (2001). La incidencia de abusos entre compañeros en alumnos de Primaria. En J. A. Amezcua, F. Peñafiel, y D. González (coords.), El Psicopedagogo en el Desarrollo Comunitario. La Planificación de los Recursos Humanos. Granada: Grupo Editorial Universitario.

Quiroga, M. A., y Forteza, J. A. (1988). La reflexividad-impulsividad. Estado de la cuestión y análisis de las características psicométricas del test MFF20. Investigaciones Psicologicas, 5, 97-125.

Salkind, N. J., y Wright, J. C. (1977). The development of reflectionimpulsivity and cognitive efficiency. Human Development, 20, 377387.

Schleifer, L., y Douglas, M. (1973). Moral judgements, behaviour and cognitive style in young children. Canadian Joumal of Behavioral Science, 5, 133-144.

Servera, M. (1997). Evaluación de los estilos cognitivos. En G Buela, y J. C. Sierra (Eds.), Manual de Evaluación Psicológica (pp. 683704). Madrid: Siglo XXI.

Servera, M., y Galván, M. R. (2001). Problemas de Impulsividad e Inatención en el Niño. Propuestas para su Evaluación. Madrid: MECD.

Vallés, M. S. (2000). Técnicas Cualitativas de Investigación Social. Reflexión Metodológica y Práctica Profesional. Madrid: Síntesis. 
Vieira, M., Fernández, I., y Quevedo, G (1989). Violence, bullying and counselling in the Iberican Peninsula. En E. Roland, y E. Munthe (Eds.), Bullying: An Intemational Perspective (pp. 223-245). London: David Fulton Publishers.

Ward, W. (1973). Reflection-impulsivity in kindergarten children. Child Development, 39, 867-874.

Weintraub, S. A. (1973). Self-control as a correlate of an internalizingexternalizing symptom dimesion. Journal of Abnormal Child Psychology, 1, 292-307.

Whitney, I., y Smith, P. K. (1993). A survey of the nature and the extent of bullying in junior, middle and secondary schools. Educational Research, 35, 3-25.

Zakay, D., Bar-El, Z., y Kreiter, S. (1984). Cognitive orientation and changing the impulsivity of children. British Joumal of Educational Psychology, 54, 40-50.

Zelniker, T., y Jeffrey, W. E. (1976). Reflective and impulsive children strategies of information processing underlying differences in problem solving. Monographs of the Society for Research in Child Development, 41(5), 1-59. 